\title{
STRONG UNIQUE CONTINUATION PROPERTY FOR ELLIPTIC SYSTEMS OF NORMAL TYPE IN TWO INDEPENDENT VARIABLES
}

\author{
Dedicated to Professor Norio Shimakura on his sixtieth birthday
}

\author{
TAKASHI ŌKAJI
}

(Received June 28, 2000, revised April 9, 2001)

\begin{abstract}
We give a result on strong unique continuation property for a certain elliptic system of first order in the two dimensional space. Two coefficient matrices are normal and commutative with each other. We assume, further, that their components are Hölder continuous and have continuous first order derivatives except at one point. Without any regularity assumptions on the eigenvalues, we can show the strong unique continuation property for a class of such systems under certain quantitative conditions on the first order derivatives. This result gives an improvement of a work by G. N. Hile and M. H. Protter in a special case.
\end{abstract}

1. Introduction. In [4], Hile and Protter obtained an interesting result on unique continuation property for a class of elliptic systems in two independent variables. Let $\Omega$ be a nonempty open connected subset of $\boldsymbol{R}^{2}$. Without loss of generality, we may assume that it contains the origin. They considered a system of the form

$$
\left|u_{x}+N(x, y) u_{y}\right| \leq M|u|, \quad(x, y) \in \Omega,
$$

where $N$ is an $m \times m$ matrix with complex entries of class $C^{1}(\Omega)$ and $M$ is a constant.

They proved there, roughly speaking, that if $N$ is a normal elliptic matrix, any solution of (1.1), satisfying

$$
\lim _{r \rightarrow 0}\left(\exp \left(x^{2}+y^{2}\right)^{-\beta / 2}\right) u(x, y)=0 \text { for all } \beta>0
$$

vanishes in $\Omega$ (Theorem 2 in [4]).

Unfortunately, their assumption (1.2) that the solution must vanish of exponentially order at the origin is too restrictive, at least, in a certain case. Indeed, we can show that if there exists a non-real complex number $\zeta$ such that all the eigenvalues of $N(0,0)$ are equal to either $\zeta$ or $\bar{\zeta}$, the function $u \in C^{1}$ that satisfies such systems and vanishes of infinite order at the origin is identically zero. In addition, we can treat non bounded potentials.

We emphasis that there is no regularity assumptions on the eigenvalues of $N$ in our work as well as in [4]. This prevents us to use a usual smooth diagonalization approach, employed by Carleman [1] and Douglis [2]. To overcome these difficulties, we shall use a technique developed in our early works for the Dirac or Maxwell equations ([3] and [5]).

2000 Mathematics Subject Classification. Primary 35B05; Secondary 35J45.

Key words and phrases. Strong unique continuation, Elliptic system. 
2. Statement of result. Let $\Omega$ be a nonempty open connected subset of $\boldsymbol{R}^{2}$ containing the origin. We define $\dot{\Omega}=\Omega \backslash\{0\}$. We denote by $r$ the distance between $(x, y)$ and the origin. $\mathcal{B}^{1, \kappa}(\Omega)$ denotes the class of functions $f$ defined on $\Omega$ satisfying that $f$ is Hölder continuous of order $\kappa$

$$
\left|f(X)-f\left(X^{\prime}\right)\right| \leq C\left|X-X^{\prime}\right|^{\kappa} \quad \text { for all } X, X^{\prime} \in \Omega,
$$

and it is continuously differentiable in $\dot{\Omega}$ such that

$$
\lim _{\rho \rightarrow 0} \sup _{0<r \leq \rho}\left\{r\left|f_{x}(x, y)\right|+r\left|f_{y}(x, y)\right|\right\}=0 .
$$

We consider the system of differential operators

$$
A(x, y) \partial_{x}+B(x, y) \partial_{y},
$$

where $A$ and $B$ are $m \times m$ normal matrices defined in $\Omega$. Further, we assume that they commute with each other, and either $A$ or $B$ is invertible at any point of $\Omega$. Thus, locally, it is equivalent to the following system.

$$
L u=\partial_{x} u+N(x, y) \partial_{y} u,
$$

where $N(x, y)$ is also an $m \times m$ normal matrix and $u$ is a function on $\Omega$ with range in $C^{m}$.

Throughout this paper, we shall assume the following properties (2.1), (2.2) and (2.3):

$$
\begin{gathered}
N(x, y) \in \mathcal{B}^{1, \kappa}(\Omega) . \\
N^{*} N=N N^{*} \text { on } \Omega,
\end{gathered}
$$

where $N^{*}$ is the conjugate transposed matrix of $N$. Let $\lambda_{j}, j=1,2, \ldots m$, be the eigenvalues of $N$. Then there exists a positive number $\delta$ such that

$$
\left|\operatorname{Im} \lambda_{j}(x, y)\right| \geq \delta, \quad j=1,2, \ldots m
$$

for all $(x, y) \in \Omega$. We write them as

$$
\lambda_{j}=\mu_{j}+i v_{j}, \quad \mu_{j}, \mu_{j} \in \boldsymbol{R} .
$$

If all the eigenvalues of $N(0,0)$ are simple, we can smoothly diagonalize $N(x, y)$ near the origin. In this case, the equation $L u=0$ is equivalent to a family of first order single equations. On the other hand, if $N(0,0)$ has multiple eigenvalues, there is no smooth diagonalization of $N(x, y)$ in general. In particular, we shall treat the case when there exists a nonreal complex number $\zeta$ such that for each $j=1, \ldots m$,

$$
\lambda_{j}(0,0)=\zeta \quad \text { or } \bar{\zeta} .
$$

Define the positive number $M_{0}$ as

$$
M_{0}^{2}=\max _{(x, y) \in S^{1}}\left(x^{2}+(\operatorname{Im} \zeta)^{-2}(\operatorname{Re} \zeta x+y)^{2}\right),
$$

where $\boldsymbol{S}^{1}$ denotes the unit sphere $\left\{(x, y) \in \boldsymbol{R}^{2} ; x^{2}+y^{2}=1\right\}$. We say that $u \in L^{2}(\Omega)$ vanishes of infinite order at the origin if

$$
\lim _{R \rightarrow 0} R^{-N} \int_{r \leq R}|u|^{2} d x d y=0 \text { for all } N>0
$$


TheOrem 2.1. Suppose (2.1)-(2.4). Let $u \in H_{\mathrm{loc}}^{1}\left(\Omega ; \boldsymbol{C}^{m}\right)$ satisfy

$$
|L u| \leq K_{0}|u| / r \quad \text { on } \dot{\Omega} .
$$

If $K_{0}<\left(2 M_{0}\right)^{-1}$ and $u$ vanishes of infinite order at the origin, then $u$ is identically zero in $\Omega$.

REMARK 2.1. When $m=1$, Pan showed that the conclusion holds for any large $K_{0}$ (Lemma 7 in [6]).

To prove Theorem 2.1, we shall use two types of Carleman inequalities. Let

$$
R(x, y)=\left\{x^{2}+(\operatorname{Im} \zeta)^{-2}(-\operatorname{Re} \zeta x+y)^{2}\right\}^{1 / 2} .
$$

First of all, we shall derive a Carleman inequality with some remainder terms.

THEOREM 2.2. For an arbitrary small positive number $\varepsilon$, there exists a positive constant $C$ such that

$$
\begin{aligned}
\frac{1}{4} \int R^{-2 \gamma-2}|u|^{2} d x d y \leq & (1+\varepsilon) \int R^{-2 \gamma}|L u|^{2} d x d y \\
& +C\left(1+\varepsilon^{-1}\right) \int R^{-2 \gamma+2 \kappa}\left|\partial_{y} u\right| d x d y
\end{aligned}
$$

for any $u(x, y) \in C_{0}^{1}\left(\dot{\Omega} ; \boldsymbol{C}^{m}\right)$ and any $\gamma \in N+1 / 2$.

As a direct consequence of Theorem 2.2 and the ellipticity of $L$, we have

THEOREM 2.3. Suppose that $K_{0}<\left(2 M_{0}\right)^{-1}$. If u satisfying (2.6) vanishes of infinite order at the origin, then there exist positive constants $B$ and $C$ such that

$$
\int_{0 \leq R(x, y) \leq \rho}\left\{|u|^{2}+\left|\partial_{x} u\right|^{2}+\left|\partial_{y} u\right|^{2}\right\} d x d y \leq C \exp \left(-B \rho^{-\kappa}\right)
$$

for any small positive $\rho$.

For the sake of Theorem 2.3, we have

$$
\int R^{-3}|\log R| \exp \left\{\gamma(\log R)^{2}\right\}\left\{|u|^{2}+\left|u_{x}\right|^{2}+\left|u_{y}\right|^{2}\right\} d x d y<\infty .
$$

Thus, we can use another Carleman inequality with a stronger weight function.

THEOREM 2.4. For a sufficiently small $\Omega$, we have

$\gamma \int R^{-2}|\log R| \exp \left\{\gamma(\log R)^{2}\right\}|u|^{2} R^{-1} d x d y \leq C \int \exp \left\{\gamma(\log R)^{2}\right\}|L u|^{2} R^{-1} d x d y$ for any $u(x, y) \in C_{0}^{1}\left(\dot{\Omega} ; \boldsymbol{C}^{m}\right)$ and any large positive $\gamma$.

Theorem 2.1 follows from Theorems 2.4 and 2.3 by the standard procedure.

3. Systems of operators with constant matrices. The proof of Theorem 2.2 is based on a perturbation argument for systems of operators with constant coefficients. Define $N_{0}=$ 
$N(0,0)$. We consider

$$
L_{0} u=u_{x}+N_{0} u_{y} .
$$

Then the first result we will show is the Carleman estimate for $L_{0}$.

THEOREM 3.1.

$$
\frac{1}{4} \int R^{-2 \gamma-2}|u|^{2} d x d y \leq \int R^{-2 \gamma}\left|L_{0} u\right|^{2} d x d y
$$

for any $u \in C_{0}\left(\dot{\Omega} ; \boldsymbol{C}^{m}\right)$ and any $\gamma \in N+1 / 2$.

ProOF. By the assumptions (2.2), (2.3) and (2.4), it follows that one can find a unitary matrix $U$ such that

$$
U^{-1} N_{0} U=\zeta I \oplus \bar{\zeta} I,
$$

where $\zeta=\lambda_{1}(0,0)=\mu+i v, \mu, v \in \boldsymbol{R}$ satisfying $|\nu|=\delta>0$. Then, $U^{-1} L_{0} U$ is also a diagonal operator with components

$$
\Lambda_{+}=\partial_{x}+(\mu+i v) \partial_{y} \quad \text { or } \quad \Lambda_{-}=\partial_{x}+(\mu-i v) \partial_{y} .
$$

We make a change of variables:

$$
\xi=x, \quad \eta=v^{-1}(-\mu x+y) .
$$

The operators $\Lambda$ and $\bar{\Lambda}$ are transformed respectively into the following operators:

$$
P_{+}=\partial_{\xi}+i \partial_{\eta} \quad \text { and } \quad P_{-}=\partial_{\xi}-i \partial_{\eta}
$$

In what follows, $\dot{\tilde{\Omega}}$ denotes the image of $\dot{\Omega}$ under the map (3.2).

Lemma 3.2. Let $*$ be either + or - . Then,

$$
\frac{1}{4} \int r^{-2 \gamma-2}|u|^{2} d \xi d \eta \leq \int r^{-2 \gamma}\left|P_{*} u\right|^{2} d \xi d \eta
$$

for any $u \in C_{0}^{1}(\dot{\tilde{\Omega}})$ and any $\gamma \in N+1 / 2$.

Proof. Introduce the polar coordinates $(\xi, \eta)=r \omega, \omega=(\cos \theta, \sin \theta) \in S^{1}$. Thus,

$$
P_{+}=\left(\omega_{1}+i \omega_{2}\right)\left(\partial_{r}+i \frac{1}{r} \partial_{\theta}\right) .
$$

Making the change of variables $z=\log r$, we see that

$$
r\left(\omega_{1}-i \omega_{2}\right) P_{+}=\partial_{z}+i \partial_{\theta} .
$$

We use the Fourier series expansion of $u(z, \cdot) \in L^{2}\left(\boldsymbol{S}^{1}\right)$ :

$$
u(z, \theta)=\sum_{k \in \boldsymbol{Z}} u_{k}(z) e^{i k \theta}, \quad \int_{0}^{2 \pi}|u(z, \theta)|^{2} d \theta=2 \pi \sum_{k \in \boldsymbol{Z}}\left|u_{k}(z)\right|^{2} .
$$

Then,

$$
\left(\partial_{z}+i \partial_{\theta}\right) u(z, \theta)=\sum_{k \in Z}\left(\partial_{z}-k\right) u_{k}(z) e^{i k \theta} .
$$


Since

$$
\begin{aligned}
\int r^{-2 \gamma^{\prime}}\left|P_{+} u\right|^{2} d \xi d \eta & =\int\left|e^{z} P_{+} u\right|^{2} e^{-2 \gamma^{\prime} z} d z d \theta \\
& =\int\left|\left(\partial_{z}+i \partial_{\theta}\right) u(z, \theta)\right|^{2} e^{-2 \gamma^{\prime} z} d z d \theta,
\end{aligned}
$$

an integration by parts gives

$$
\begin{aligned}
& \int r^{-2 \gamma}\left|P_{+} u\right|^{2} d \xi d \eta \\
& \quad=\sum_{k \in \mathbf{Z}} \int\left|\partial_{z}\left(e^{-\gamma z} u_{k}\right)\right|^{2} d z d \theta+\sum_{k \in \boldsymbol{Z}} \int\left|(\gamma-k) e^{-\gamma z} u_{k}\right|^{2} d z d \theta .
\end{aligned}
$$

We choose $\gamma \in N+1 / 2$. Then, from (3.4) and (3.6), we arrive at the inequality (3.3) with $\gamma \in N+1 / 2$. In the same way, we obtain the assertion for $P_{-}$.

As a result, we obtain the following

Proposition 3.3. Let $*$ be either + or - . Then,

$$
\frac{1}{4} \int R^{-2 \gamma-2}|u|^{2} d x d y \leq \int R^{-2 \gamma}\left|\Lambda_{*} u\right|^{2} d x d y
$$

for any $u(x, y) \in C_{0}^{1}(\dot{\Omega})$ and any $\gamma \in N+1 / 2$.

Theorem 3.1 is a direct consequence of Proposition 3.3.

Now, we proceed to the proof of Theorem 2.2. We can write

$$
L=L_{0}+L_{1}, \quad L_{1}=\tilde{N} \partial_{y},
$$

where $\tilde{N}=N(x, y)-N(0,0)$ has its entries satisfying

$$
\left|\tilde{N}_{i j}(x, y)\right| \leq C r^{\kappa},
$$

because of their Hölder continuity. We use the inequality

$$
(a+b)^{2} \leq(1+\varepsilon) a^{2}+\left(1+\varepsilon^{-1}\right) b^{2} .
$$

This observation together with Theorem 3.1 leads to the Carleman inequality with a remainder term in Theorem 2.2.

4. Proof of Theorem 2.3. Now, we turn to the proof of Theorem 2.3. We require the following elliptic estimate.

Lemma 4.1. There exists a positive constant $C_{1}$ such that for any $f \in C_{0}^{1}\left(\Omega ; \boldsymbol{C}^{m}\right)$,

$$
\int\left(\left|\partial_{x} f\right|^{2}+\left|\partial_{y} f\right|^{2}\right) d x d y \leq C_{1} \int\left(|L f|^{2}+|f|^{2}\right) d x d y .
$$

PROOF. This can be easily verified if we use a partition of unity to reduce the problem for a finite number of constant matrices $\left\{N\left(x_{j}, y_{j}\right)\right\}_{j=1}^{N}$ in the standard manner. 
Let $\chi_{0}$ be a nonnegative smooth function whose support is compact and contained in $U \subset \Omega$ such that $\chi_{0}(x, y)=1$ near the origin. Let $u \in H_{\text {loc }}^{1}\left(\Omega ; \boldsymbol{C}^{m}\right)$ satisfy (1.1). We note that the inequality (4.1) holds when $f \in H_{0}^{1}\left(\Omega ; \boldsymbol{C}^{m}\right)$. If we apply (4.1) to $f(x, y)=$ $R(x, y)^{-\gamma} \chi_{0}(x, y) u(x, y)$, we see that there exists a positive constant $K$ such that

$$
\gamma^{-2} \int_{B\left(r_{0}\right)} R^{-2 \gamma}|\nabla u|^{2} d x d y \leq K \gamma^{-2} \int_{U} R^{-2 \gamma}|L u|^{2} d x d y+K \int_{U} R^{-2 \gamma-2}|u|^{2} d x d y
$$

and

$$
\begin{aligned}
& \gamma^{-2} \int_{B\left(r_{0}\right)} R^{-2 \gamma+2 \kappa}\left|\partial_{y} u\right|^{2} d x d y \\
& \quad \leq K \gamma^{-2} \int_{U} R^{-2 \gamma+2 \kappa}|L u|^{2} d x d y+K \int_{U} R^{-2 \gamma-2+2 \kappa}|u|^{2} d x d y
\end{aligned}
$$

with some small positive number $r_{0}$. Let $\chi(r)$ be a nonnegative function belonging to $C_{0}^{1}((-\infty, 2))$ such that $\chi(r)=1$ when $0 \leqq r<1$.

In what follows, for $r>0$, let $\tilde{B}(r)=\{R(x, y) \leq r\}$. We shall consider $\tilde{u}(x, y)=$ $\chi\left(M \gamma^{1 / \kappa} R\right) u(x, y)$. Here, $M$ is a large positive parameter, which will be determined later.

We observe that

$$
x^{2}+v^{-2}(-\mu x+y)^{2} \leq M_{0}^{2}\left(x^{2}+y^{2}\right) \text { for all }(x, y) \in \boldsymbol{R}^{2},
$$

and

$$
|L u(x, y)| \leq K_{0} M_{0}|u(x, y)| / R(x, y) .
$$

Thus, combining Theorem 2.2 with (4.2) and (4.3), we see that

$$
\begin{aligned}
& \beta \int R^{-2 \gamma-2}|\tilde{u}|^{2} d x d y+\gamma^{-2} N^{-1} \int R^{-2 \gamma}|\nabla \tilde{u}|^{2} d x d y \\
& \leq K \int R^{-2 \gamma+2 \kappa}\left(|\nabla \tilde{u}|^{2}+R^{-2}|\tilde{u}|^{2}\right) d x d y \\
& \quad+C M^{2} \gamma^{2 / \kappa} \int_{M^{-1} \gamma^{-1 / \kappa} \leq R \leq 2 M^{-1} \gamma^{-1 / \kappa}} R^{-2 \gamma}|u|^{2} d x d y,
\end{aligned}
$$

where $\beta$ is the positive number satisfying

$$
\beta^{2}=\frac{1}{4}-\left\{(1+\varepsilon)\left(K_{0} M_{0}\right)^{2}+\gamma^{-2} \frac{K}{N}\right\}-\frac{K}{N}
$$

which is positive by our assumption and by taking $N$ and $\varepsilon^{-1}$ to be large enough.

Choose $M$ such that $K M^{-2 \kappa}<1 /(2 N)$. Then it holds that

$$
K R^{2 \kappa}<\frac{1}{N \gamma^{2}} \quad \text { and } \quad M^{2} \gamma^{2 / \kappa} R^{2} \leq 4
$$


if $0 \leq R \leq 2 M^{-1} \gamma^{-1 / \kappa}$. It follows that

$$
\begin{gathered}
\frac{\beta}{2} \int_{\tilde{B}\left(1 /\left(2 M \gamma^{1 / \kappa}\right)\right)} R^{-2 \gamma-2}|u|^{2} d x d y+\left(2 \gamma^{2} N\right)^{-1} \int_{\tilde{B}\left(1 /\left(2 M \gamma^{1 / \kappa}\right)\right)} R^{-2 \gamma}|\nabla u|^{2} d x d y \\
\leq C \int_{U \backslash \tilde{B}\left(1 /\left(M \gamma^{1 / \kappa}\right)\right)} R^{-2 \gamma-2}\left\{|u|^{2}+|\nabla u|^{2}\right\} d x d y,
\end{gathered}
$$

if $U$ is an arbitrary sufficiently small neighborhood of the origin. Here, we have used the inequality

$$
R^{2 \kappa} \leq \beta / 2 \quad \text { if }(x, y) \in U .
$$

From the inequality (4.5), we conclude that

$$
\begin{aligned}
& \left(2 M \gamma^{1 / \kappa}\right)^{2 \gamma+2} \int_{\tilde{B}\left(1 /\left(2 M \gamma^{1 / \kappa}\right)\right)}\left\{|u|^{2}+\left(2 \gamma^{2} N\right)^{-1}|\nabla u|^{2}\right\} d x d y \\
& \quad \leq C\left(M \gamma^{1 / \kappa}\right)^{2 \gamma+2} \int_{U \backslash \tilde{B}\left(1 /\left(M \gamma^{1 / \kappa}\right)\right)}\left\{|u|^{2}+|\nabla u|^{2}\right\} d x d y .
\end{aligned}
$$

As a result, we have

$$
\int_{\tilde{B}\left(1 /\left(2 M \gamma^{1 / \kappa}\right)\right)}\left\{|u|^{2}+\left(2 \gamma^{2} N\right)^{-1}|\nabla u|^{2}\right\} d x d y \leq C 2^{-2 \gamma-2} \int_{U}\left\{|u|^{2}+|\nabla u|^{2}\right\} d x d y
$$

for any large positive $\gamma \in N+1 / 2$. This leads to the desired conclusion of Theorem 2.3.

5. Proof of Theorem 2.4. As in the proof of Lemma 3.1, we can find a unitary transformation and a change of variables (3.2) such that

$$
L=\partial_{x}+N \partial_{y}, \quad N(0,0)=i I \oplus(-i) I
$$

and

$$
|L u| \leq K_{0} M_{0}|u| / r .
$$

We see that $L$ is written in the polar coordinates as

$$
\left(\omega_{1}+N \omega_{2}\right) \partial_{r}+r^{-1}\left(-\omega_{2}+N \omega_{1}\right) \partial_{\theta} .
$$

We multiply the above operator by the cofactor ${ }^{\mathrm{co}} J$ of $J=\left(\omega_{1}+N \omega_{2}\right)$ from its left. It holds that

where

$$
{ }^{\mathrm{co}} J L=\operatorname{det} J \partial_{r}+\frac{1}{r} G(r, \omega) \partial_{\theta},
$$

$$
G={ }^{\mathrm{co}} J\left(-\omega_{2}+\omega_{1} N\right) .
$$

LEMMA 5.1. G is also a normal matrix with eigenvalues $\kappa_{j}$ satisfying

$$
\left|\operatorname{Im} \kappa_{j}\right| \geq \delta^{\prime}, \quad j=1, \ldots, m .
$$

Proof. Let $r \geq 0$ and $\omega \in S^{1}$ satisfy $r \omega \in \Omega$. Then, there exists an orthogonal matrix $T$ such that

$$
N=T^{-1} D T, \quad D=\operatorname{diag}\left(\lambda_{1}, \ldots, \lambda_{m}\right) .
$$


Furtheremore,

$$
{ }^{\text {co }} J=(\operatorname{det} J) T^{-1}\left(\omega_{1} I+\omega_{2} D\right) T
$$

and

$$
-\omega_{2}+\omega_{1} N=T^{-1}\left(-\omega_{2} I+\omega_{1} D\right) T,
$$

so that $T G T^{-1}$ is a diagonal matrix with componets given by

$$
\kappa_{j}=\operatorname{det} J\left(\omega_{1}+\omega_{2} \lambda_{j}\right)^{-1}\left(-\omega_{2}+\omega_{1} \lambda_{j}\right) .
$$

$$
\begin{gathered}
\text { Let } \varphi=\gamma\left(\log \sqrt{x^{2}+y^{2}}\right)^{2} / 2, e^{\varphi} u=v, \tilde{L}=(\operatorname{det} J)^{-1 \operatorname{co} J L \text { and }} \\
e^{\varphi} \tilde{L} u=L_{\varphi} v .
\end{gathered}
$$

Then, we see that

$$
L_{\varphi} v=\left\{\partial_{r}-\varphi^{\prime}\right\} v+\frac{1}{r}(\operatorname{det} J)^{-1} G \partial_{\theta} v .
$$

Let $S=(\operatorname{det} J)^{-1}\left(G+G^{*}\right) / 2$ and $Q=(\operatorname{det} J)^{-1}\left(G-G^{*}\right) /(2 i)$. We have to consider

$$
\begin{aligned}
& \int\left|L_{\varphi} v\right|^{2} r^{-1} d x d y \\
& =\int\left|\partial_{r} v+\frac{1}{r} S \partial_{\theta} v+\frac{1}{2 r}\left(\partial_{\theta} S\right) v\right|^{2} r^{-1} d x d y \\
& \quad+\int\left|-\varphi^{\prime} v+\frac{i}{r} Q \partial_{\theta} v-\frac{1}{2 r}\left(\partial_{\theta} S\right) v\right|^{2} r^{-1} d x d y \\
& \quad+2 \operatorname{Re} \int\left(\partial_{r} v+\frac{1}{r} S \partial_{\theta} v+\frac{1}{2 r}\left(\partial_{\theta} S\right) v,-\varphi^{\prime} v+\frac{i}{r} Q \partial_{\theta} v-\frac{1}{2 r}\left(\partial_{\theta} S\right) v\right) r^{-1} d x d y .
\end{aligned}
$$

By an integration by parts, it follows that

$$
2 \operatorname{Re} \int\left(\frac{1}{r} S \partial_{\theta} v+\frac{1}{2 r}\left(\partial_{\theta} S\right) v, \varphi^{\prime}(r) v\right) r^{-1} d x d y=0
$$

and

$$
2 \operatorname{Re} \int\left(\partial_{r} v,-\varphi^{\prime} v\right) r^{-1} d x d y=\int \varphi^{\prime \prime}|v|^{2} r^{-1} d x d y
$$

We shall use the relation

$$
|\nabla v|^{2}=\left|\partial_{r} v\right|^{2}+\frac{1}{r^{2}}\left|\partial_{\theta} v\right|^{2}
$$

For a positve scalar function $f(x, y)$ defined in $\dot{\Omega}$, we shall use the notation $u(x, y)=$ $o(f(x, y))$ if a scalar function $u(x, y)$ defined in $\Omega$ satisfies

$$
\lim _{\rho \rightarrow 0} \sup _{0<r \leq \rho}\left|\frac{u(x, y)}{f(x, y)}\right|=0 \text {. }
$$

Since $Q S=S Q$ at each point of $\Omega$ and

$$
|\nabla Q|+|\nabla S|=o(1 / r)
$$


the relations (5.2) and (5.3) imply that

$$
\begin{aligned}
& 2 \operatorname{Re} \int\left(\partial_{r} v+\frac{1}{r} S \partial_{\theta} v+\frac{1}{2 r}\left(\partial_{\theta} S\right) v,-\varphi^{\prime} v+\frac{i}{r} Q \partial_{\theta} v-\frac{1}{2 r}\left(\partial_{\theta} S\right) v\right) r^{-1} d x d y \\
& \geq \int \varphi^{\prime \prime}|v|^{2} r^{-1} d x d y-\int o\left(r^{-1}\right)\left(r^{-1}|v|\left|\partial_{r} v\right|+r^{-1}|v|\left|r^{-1} v_{\theta}\right|\right) r^{-1} d x d y .
\end{aligned}
$$

Therefore, we conclude that for any $\varepsilon>0$, there exists a neighborhood $U$ of the origin such that

$$
\begin{aligned}
\int \varphi^{\prime \prime}|v|^{2} r^{-1} d x d y \leq & C \int\left|L_{\varphi} v\right|^{2} r^{-1} d x d y \\
& +\varepsilon \int\left(r^{-1}|v|\left|\partial_{r} v\right|+r^{-1}|v|\left|r^{-1} v_{\theta}\right|\right) r^{-1} d x d y
\end{aligned}
$$

for every $v \in C_{0}^{1}(U)$. Note that

$$
\varphi^{\prime \prime}=-\gamma r^{-2} \log r+\gamma r^{-2}
$$

and there exists a positive constant $C$ such that

$$
\begin{aligned}
& \int\left\{r^{-1}|v|\left|v_{r}\right|+r^{-2}|v|\left|v_{\theta}\right|\right\} r^{-1} d x d y \\
& \leq \int\left|r^{-1} v\right|\left|\left(\partial_{r}+r^{-1}\right) v+\frac{1}{r} S \partial_{\theta} v+\frac{1}{2 r}\left(\partial_{\theta} S\right) v\right| r^{-1} d x d y \\
& \quad+C\left\{\int\left|r^{-1} v\right|\left|r^{-1} \partial_{\theta} v\right| r^{-1} d x d y+\int r^{-2}|v|^{2} r^{-1} d x d y\right\}
\end{aligned}
$$

and

$$
\begin{aligned}
& \int\left|r^{-1} v \| r^{-1} \partial_{\theta} v\right| r^{-1} d x d y \\
& \quad \leq C\left\{\gamma \int r^{-2}|\log r||v|^{2} r^{-1} d x d y+\gamma^{-1} \int|\log r|^{-1}|\nabla v|^{2} r^{-1} d x d y\right\} .
\end{aligned}
$$

On the other hand, putting $v=e^{\varphi} u$ into (4.1), we obtain that

$$
\begin{aligned}
& \gamma^{-1} \int|\log r|^{-1}|\nabla v|^{2} r^{-1} d x d y \\
& \quad \leq C \int e^{2 \varphi}|\tilde{L} u|^{2} r^{-1} d x d y+C \gamma \int r^{-2}|\log r| e^{2 \varphi}|u|^{2} r^{-1} d x d y
\end{aligned}
$$

because

We shall use

$$
L_{\varphi} v=e^{\varphi} \tilde{L} u+\mathcal{O}\left(\varphi^{\prime}\right) u, \quad \varphi^{\prime}=\gamma r^{-1} \log r .
$$

$$
\int r^{-2}|v|^{2} r^{-1} d x d y=\int o(1) r^{-2}|\log r||v|^{2} r^{-1} d x d y .
$$

From (5.5), (5.6) and (5.7), we obtain the desired estimate in Theorem 2.4 if $\Omega$ is sufficiently small. 
6. Proof of Theorem 2.1. Let $V(r)=\{(x, y) ; R(x, y) \leq r\}$ for each $r>0$. Suppose that $\sigma>0$ is sufficiently small so that Theorem 2.4 holds for $\Omega=V(\sigma)$. Let $u \in H_{\text {loc }}^{1}\left(V(\sigma) ; C^{m}\right)$ satisfy (2.6), and let $\chi \in C_{0}^{\infty}(\Omega), 0 \leq \chi \leq 1$ be a cut-off function such that $\chi(x, y)=1$ if $R(x, y) \leq \sigma / 3$ and $\chi=0$ if $R(x, y) \geq \sigma / 2$. By taking a limiting procedure, in view of Theorem 2.3, we can apply Theorem 2.4 to $\tilde{u}=\chi(x, y) u(x, y)$. Let $\varphi(x, y)=\gamma|\log R(x, y)|^{2} / 2$. It holds that there exists a positive constant $C$ such that

$$
\begin{gathered}
\gamma \int R^{-3}|\log R| e^{2 \varphi}|\tilde{u}|^{2} d x d y \leq C \int e^{2 \varphi}|L \tilde{u}|^{2} R^{-1} d x d y, \\
\int_{\Omega} e^{2 \varphi}|[L, \chi] u|^{2} R^{-1} d x d y \leq C \int_{\Omega \backslash V(\sigma / 3)} e^{2 \varphi}|u|^{2} R^{-1} d x d y .
\end{gathered}
$$

Thus,

$$
\gamma \int_{V(\sigma / 4)} e^{2 \varphi}|R|^{-3}|\log | R|||u|^{2} d x d y \leq C^{\prime} \int_{\Omega \backslash V(\sigma / 3)} e^{2 \varphi}|u|^{2} R^{-1} d x d y .
$$

Since $(\log r)^{2}$ is a strictly decreasing function, we have

$$
\gamma(\kappa / 4)^{-3}|\log \sigma / 4| \int_{V(\sigma / 4)}|u|^{2} d x d y \leq C^{\prime \prime} e^{\gamma\left\{(\log (\sigma / 3))^{2}-(\log (\sigma / 4))^{2}\right\}} .
$$

Thus, letting $\gamma \rightarrow \infty$, we conclude that $u=0$ in $V(\sigma / 4)$. This completes the proof of Theorem 2.1.

\section{REFERENCES}

[1] T. CARLEMAN, Sur un problèm d'unicité pour les systemes d'équations aux dérivées partielles à deux variables indépendentes, Ark. Mat., Astr. Fys. 26B (1939), 1-9.

[2] A. Douglis, Uniqueness in Cauchy problems for elliptic systems of equations, Comm. Pure Appl. Math. 6 (1953), 291-298.

[3] L. De CARLi and T. OKajI, Strong unique continuation property for the Dirac equation, Res. Inst. Math. Sci. 35 (1999), 825-846.

[4] G. N. Hile And M. H. Protter, Unique continuation and the Cauchy problem for first order systems of partial differential equations, Comm. Partial Differential Equations 1 (1976), 437-465.

[5] T. OKAJI, Strong unique continuation property for time harmonic Maxwell equations, J. Math. Soc. Japan. 54 (2002), 89-122.

[6] Y. F. PAN, Unique continuation for Schrödinger operators with singular potentials, Comm. Partial Differential Equations 17 (1992), 953-965.

DiVISION OF MATHEMATICS

GRADUATE SCHOOL OF SCIENCE

KYOTO UNIVERSITY

КYOTO, 606-8502

JAPAN 